\title{
Real-time in situ monitoring of particle and structure evolution in mechanochemical synthesis of UiO-66 metal-organic framework
}

\author{
Luzia S. Germann, ${ }^{*}$ a Athanassios D. Katsenis, ${ }^{b}$ Igor Huskić, ${ }^{b}$ Patrick A. Julien, ${ }^{b}$ Krunoslav \\ Užarević, ${ }^{c}$ Martin Etter, ${ }^{d}$ Omar K. Farha, ${ }^{e}$ Tomislav Friščić ${ }^{\star, b}$ and Robert E. Dinnebier ${ }^{\star, a}$ \\ a Max Planck Institute for Solid State Research, Heisenbergstr. 1, 70569 Stuttgart, Germany \\ b Department of Chemistry, McGill University, 801 Sherbrooke St. W., H3A 0B8, Montréal, Canada \\ ${ }^{c}$ Ruđer Bošković Institute, 10000 Zagreb, Croatia \\ d Deutsches Elektronen-Synchrotron (DESY), 22607 Hamburg, Germany \\ e Department of Chemistry, Northwestern University, Evanston, 60208 Illinois, United States \\ Corresponding authors, Luzia. S. Germann (Luzia.germann@gmail.com), Tomislav Friščić \\ (tomislav.friscic@mcgill.ca), and Robert E. Dinnebier (r.dinnebier@fkf.mpg.de)
}

\begin{abstract}
While in situ synchrotron X-ray powder diffraction has revolutionized mechanistic studies of mechanochemistry and inspired the development for other real-time monitoring approaches, the full potential of X-ray diffraction data remains unexploited. Focusing on UiO-type metal-organic frameworks (MOFs) as models, we show how in-depth analysis of real-time diffraction data can provide insight into the evolution of crystallite size and structure, including a strong influence of liquid additives on early stages of reactions.
\end{abstract}

The growing application of mechanochemistry to molecular and materials syntheses has inspired the emergence of increasingly sophisticated tools for real-time observation of mechanochemical reactions. ${ }^{1-5}$ Examples of these include tandem reaction monitoring by Raman spectroscopy and X-ray powder diffraction (XRPD), ${ }^{6-10}$ or combined XRPD and thermal measurements. ${ }^{7,8,11}$ However, XRPD alone offers a plethora of information that has not yet been exploited in reports of in situ studies, which usually stay limited to qualitative analysis of product formation, neglecting structural and microstructural changes, such as evaluation of the evolution of lattice parameter and crystallite size. ${ }^{12,13}$

Here, using as a model reaction the mechanochemical synthesis of a higly stable UiO-type metal-organic framework (MOF), we provide the first detailed, real-time analysis of the product structure and particle size during mechanosynthesis, revealing a previously not reported effect of a liquid additive on the early stage of the reaction. ${ }^{14,15}$ We chose as our model UiO-66, ${ }^{16-18}$ a fcu-topology MOF based on hexanuclear zirconium hydroxo-clusters as nodes and terephthalate as linkers, due to its demonstrated stability ${ }^{17}$ to a variety of conditions. ${ }^{19}$ We have previously shown that microporous UiO-66 and its isostructural amino-derivative UiO$66-\mathrm{NH}_{2}$ can be readily synthesized by liquid-assisted grinding (LAG), a mechanochemical technique in which the reactants are milled in the presence of a small amount of a liquid additive, of pre-assembled zirconium clusters with either terephthalic or 2-aminoterephthalic acid, respectively. ${ }^{20,21}$

We now provide a detailed analysis of the development of structure and microstructure during the LAG synthesis of UiO-66 and UiO-66- $\mathrm{NH}_{2}$ by using in situ XRPD data. All reactions were conducted by milling the pre-assembled methacrylate cluster $\mathrm{Zr}_{6} \mathrm{O}_{4}(\mathrm{OH})_{4}\left(\mathrm{C}_{2} \mathrm{H}_{3} \mathrm{CO}_{2}\right)_{12}(\mathrm{Zr6M})$ with terephthalic acid ( $\left.\mathrm{H}_{2} \mathrm{TPA}\right)$ or 2aminoterephthalic acid $\left(\mathrm{H}_{2}\right.$ TPA-NH$)$ in the respective 1:6 stoichiometric ratio. The reactions were performed using either methanol $(\mathrm{MeOH})$ or $N, N$-dimethylformamide (DMF) as the liquid additive, with the ratio of liquid 


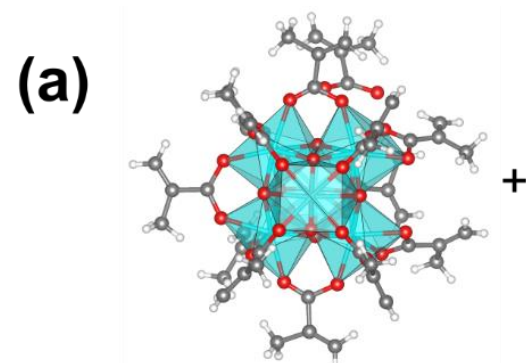

Zr6M<smiles>O=C(O)c1ccc(C(=O)O)cc1</smiles>

$\mathrm{H}_{2}$ TPA

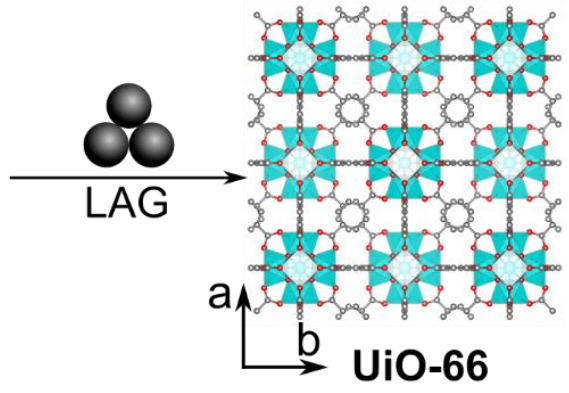

UiO-66

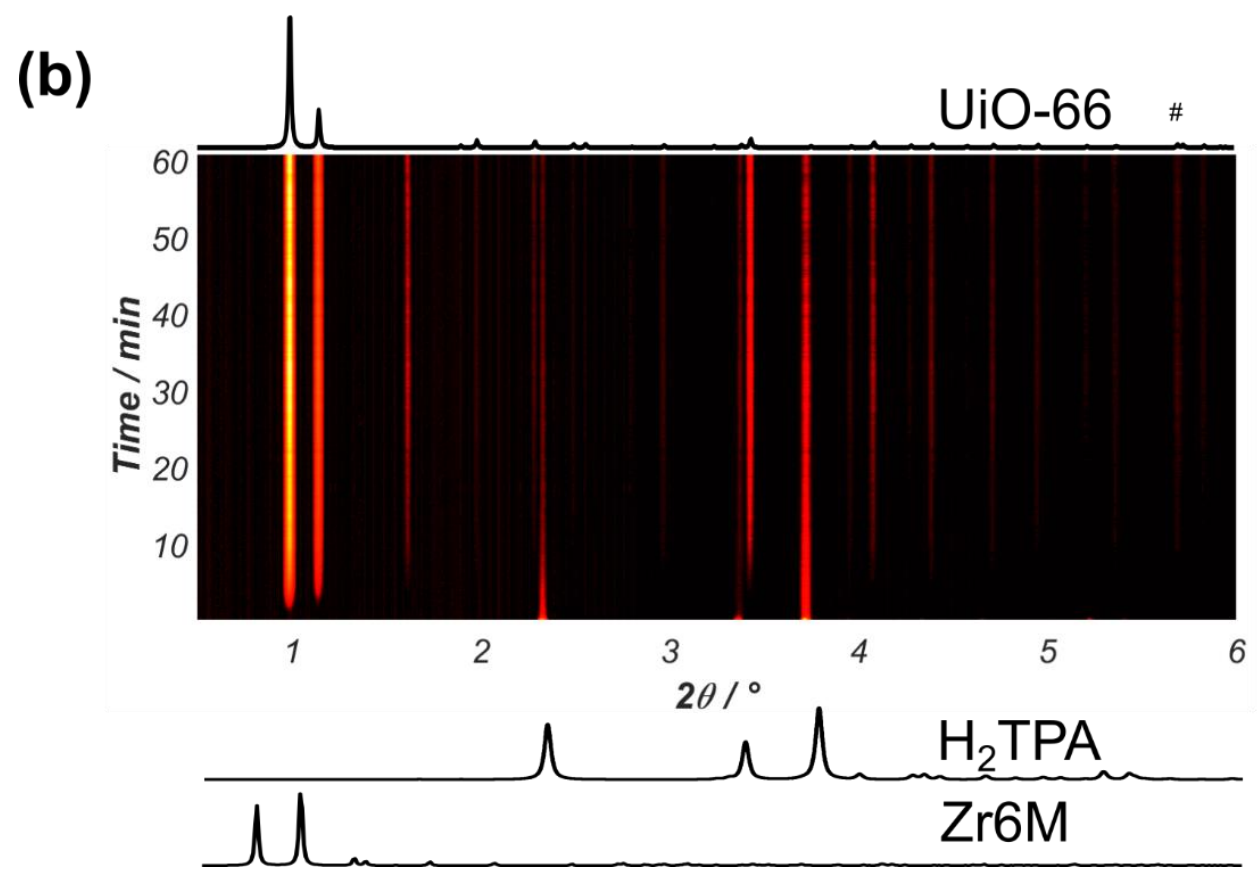

Figure 1. (a) Scheme of mechanochemical UiO-66 formation; (b) time-resolved 2D diffraction plot for the $\mathrm{LAG}$ reaction using $\mathrm{MeOH}(\eta=0.78 \mu \mathrm{L} / \mathrm{mg})$, with calculated XRPD patterns of $\mathrm{H}_{2} \mathrm{TPA}(\mathrm{CSD}$ code TEPHTH), Zr6M (CSD code REBNUH) and UiO-66 (CSD code RUBTAK) shown below and above the plot. The diffraction signal of the steel balls is marked with an \#.

volume to reactant weight $(\eta)$ in the range between $0.5 \mu \mathrm{L} / \mathrm{mg}$ and $0.78 \mu \mathrm{L} / \mathrm{mg}$ (Table 1 ). ${ }^{22}$ The reactions were performed in a poly(methylmethacrylate) (PMMA) jar mounted on a modified Retsch MM400 mill operating at $30 \mathrm{~Hz}$, using stainless steel balls (Figure 1a), and real-time monitoring was conducted at the Deutsches Elektronen-Synchrotron (DESY) P02.1 beamline using an energy of $60 \mathrm{keV}(\lambda=0.207 \AA)$.

Analysis of in situ XRPD data shows that the formation of UiO-66 by LAG with MeOH $(\eta=0.78 \mu \mathrm{L} / \mathrm{mg})$, using a single stainless steel ball of $10 \mathrm{~mm}$ diameter (4 grams weight), proceeds without any crystalline intermediates, with the diffraction signals of the MOF appearing within 2 minutes from the onset of milling (Figure 1b). A qualitative assessment of the product formation and, therefore, reaction progress was accomplished by integrating the intensity of the first, most prominent X-ray reflection $(111)$ at $2 \theta=0.988^{\circ}$. Such analysis is less sensitive to absorption of X-rays by the milling media and changes in the pore content of the nascent MOF over time. Both effects influence the absolute intensities, making Rietveld refinement challenging. Analysis of the integrated peak intensity of the (111) reflection revealed that the amount of crystalline UiO-66 rapidly increased in the first 15 minutes of milling, after which it levelled off to a steady value within ca. 40 minutes (Figure 2a). Next, we used the Scherrer method to evaluate changes in crystallite size during mechanochemical reaction. ${ }^{23}$ For this purpose the instrumental profile function (IPF) was determined with the help of a silicon standard, and subsequently deconvoluted from the measured profile. The crystallite size was obtained by taking the integral breadth of the Bragg reflections and assuming spherical particles. While microstrain could play a role in the interpretation of the data, we have opted not to include it throughout 
this analysis, since attempts at simultaneous refinement led to a high correlation between microstrain and crystallite size, with subsequent decomposition hindered due to an insufficient number of Bragg reflections. Importantly, the refinement with only crystallite size led to an equally good fit. Such analysis indicated a rapid increase of crystallite size to ca. $50 \mathrm{~nm}$ in the first 15 minutes of milling, after which the increase in crystallite size is significantly slower, reaching ca. $60 \mathrm{~nm}$ after 60 minutes (Figure 2b). To validate the results of Scherrer analysis, the reaction was repeated in a conventional laboratory setting and the particle sizes analysed by scanning electron microscopy (SEM), revealing round-shaped particles with a size range of $32-70 \mathrm{~nm}$ (Figure 2c, S13). These numbers are in good agreement with the average crystallite size obtained from the in situ XRPD analysis, validating the applicability of the Scherrer method (Figure 2b).

Table 1: Selected parameters for herein explored in situ experiments.

\begin{tabular}{|c|c|c|c|}
\hline MOF & Zr6M & LAG additive & $\eta(\mu \mathrm{L} / \mathrm{mg})$ \\
\hline UiO-66 & $161.83 \mathrm{mg}$ & $\mathrm{MeOH}, 200 \mu \mathrm{L}$ & 0.78 \\
\hline UiO-66 & $161.87 \mathrm{mg}$ & $\mathrm{MeOH}, 170 \mu \mathrm{L}$ & 0.71 \\
\hline UiO-66 & $189.13 \mathrm{mg}$. & $\mathrm{DMF}, 150 \mu \mathrm{L}$ & 0.5 \\
\hline UiO-66- $\mathrm{NH}_{2}$ & $183.17 \mathrm{mg}$ & $\mathrm{MeOH}, 200 \mu \mathrm{L}$ & 0.66 \\
\hline UiO-66-NH & $183.08 \mathrm{mg}$ & $\mathrm{DMF}, 200 \mu \mathrm{L}$ & 0.66 \\
\hline
\end{tabular}

a addition of $8.9 \mathrm{w} \%$ silicon as an internal standard

The reaction was repeated with slightly modified reaction parameters $(\eta=0.71 \mu \mathrm{L} / \mathrm{mg})$, using ca. 10 weight\% of silicon as an internal diffraction standard, with two stainless steel balls of $7 \mathrm{~mm}$ diameter as milling media (1.3 grams each). The general reaction outcome was similar as for the above-mentioned reaction. However, the reaction showed a transient induction period of about 10 minutes (Figures S1-S4 in the Electronic supporting information, ESI).

Next, we performed the reaction with DMF as the LAG additive. While this reaction also proceeded without any observable crystalline intermediates (see Figure S5), the presence of a different LAG additive slowed the reaction and led to a time delay, with the product X-ray reflections appearing only after ca. 5 minutes of milling. The integrated peak intensity of the (111) Bragg reflection of UiO-66 revealed rapid product formation within the first 10 minutes of milling, after which a plateau is reached (Figure S6). The Scherrer analysis revealed a continuous increase in crystallite size, reaching a value of approx. $10 \mathrm{~nm}$ after 55 min (Figure S7). SEM analysis of the UiO-66 materials synthesised mechanochemically by LAG with DMF revealed particles with sizes in the range of $9-20 \mathrm{~nm}$ (Figure S14), which is again in very good agreement with the average sizes obtained by Scherrer analysis of in situ XRPD data. The improved crystallinity of UiO-66 obtained by LAG with $\mathrm{MeOH}$ compared to the material obtained in presence of DMF is consistent with previous work. ${ }^{20}$

In principle, the in situ monitoring technique enables the continuous observation of not only crystallite size evolution and extent of reaction, but can also provide insights into the development of product structure. Such structural development is a complex phenomenon, potentially including changes at the level of the crystal structure, e.g. framework breathing, as well as changes in the microstructure. Breathing effects are well known from temperature dependent, high-pressure studies and from solvent uptake or release of MOFs. ${ }^{24,25}$

As the first entry into in situ investigation of such structural development upon milling, we have evaluated changes in the lattice parameter $(a)$ and unit cell volume $(V$ ) of cubic UiO-66 during its LAG synthesis. The analysis revealed a surprising difference between DMF and $\mathrm{MeOH}$ as milling liquids: the lattice parameter of nascent UiO-66 material consistently decreased in the early stage of LAG synthesis with MeOH, independent on the other experimental conditions (Figures 3a, S4). Specifically, a decreased from $20.88 \AA$ to $20.824(2) \AA$ for $\eta=0.78 \mu \mathrm{L} / \mathrm{mg}$ and to $20.805(2)$ for $\eta=0.71 \mu \mathrm{L} / \mathrm{mg}$, respectively. In contrast, LAG reactions with DMF additive reveal an initial increase in a, followed by a slight decrease, ending with a value of 20.844(4) $\AA$ for $\eta=0.5 \mu \mathrm{L} / \mathrm{mg}$ (Figure 2b). Interestingly, the UiO-66 lattice parameters observed for in situ data are slightly but significantly higher than those reported based on single crystal structure determination, and which typically 
range between $20.66 \AA$ and $20.76 \AA$ (Table S2). As the single crystal data are based on materials obtained via solution-based syntheses using $\mathrm{ZrCl}_{4}$ as the zirconium source reactant, this observation suggests that both the synthesis method (mechanochemistry or solution-based synthesis) and the choice of zirconium precursor $\left(\mathrm{ZrCl}_{4} \mathrm{Vs} \mathrm{Zr6M}\right)$ can have previously not recognized impact on the parameters of the final MOF structure.

We also explored the LAG formation of UiO-66- $\mathrm{NH}_{2}$ using either $\mathrm{MeOH}$ or DMF as liquid additives $(\eta=0.66$ $\mu \mathrm{L} / \mathrm{mg}$ ). As observed for UiO-66, milling of $\mathrm{Zr6M}$ with $\mathrm{H}_{2}$ TPA-NH $\mathrm{N}_{2}$ leads directly to the UiO-66- $\mathrm{NH}_{2}$ product
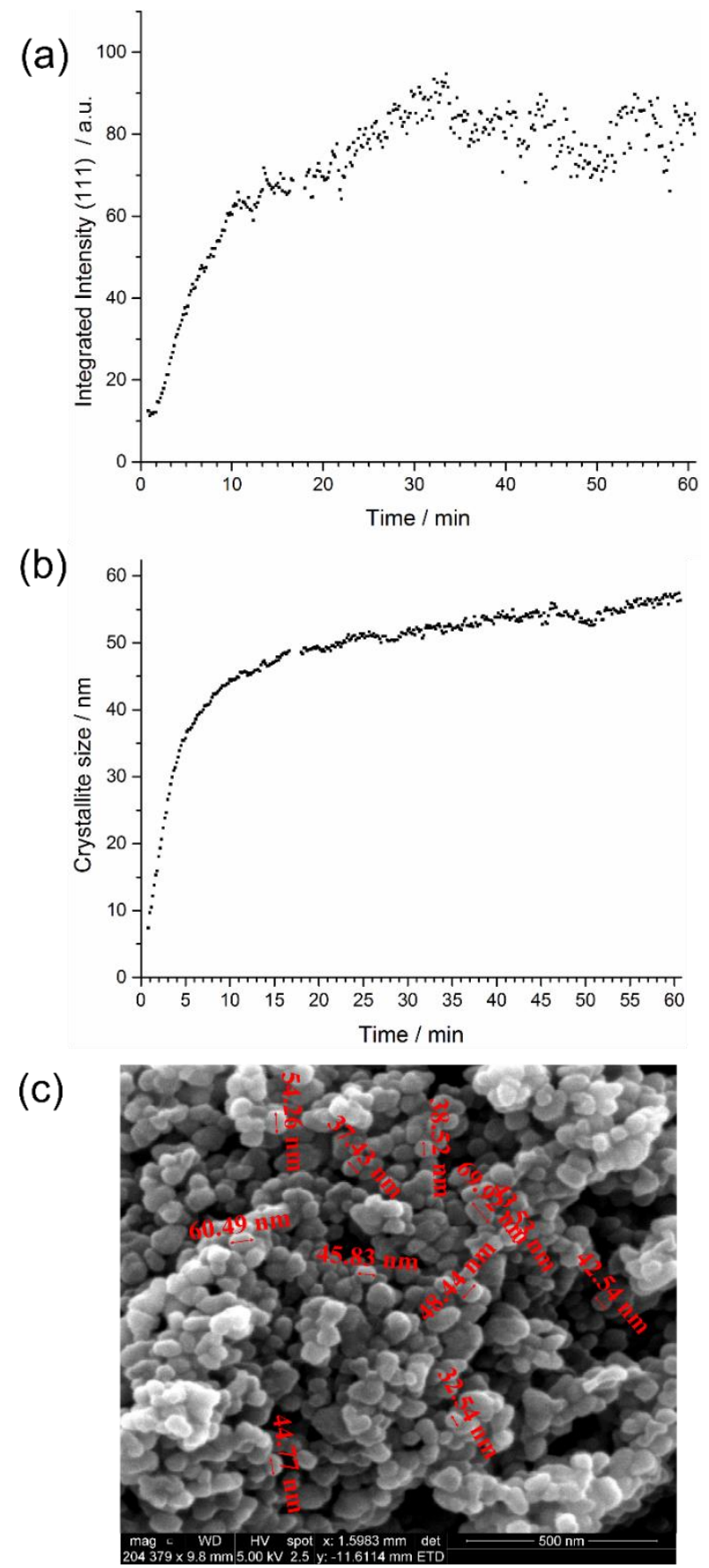

Figure 2. (a) Integrated peak intensity of the most prominent Bragg reflection (111), $2 \theta=0.98^{\circ}$, over time. (b) Evolution of particle size of UiO-66 synthesized by LAG with MeOH over of time. (c) A representative SEM image for UiO-66 synthesized using $\mathrm{MeOH}$ as $\mathrm{LAG}$ additive, $\eta=0.78 \mu \mathrm{L} / \mathrm{mg}$. 
(CSD code EWILIH) without any observable crystalline intermediate (Figures S8, S11). However, the product was found not to be as highly crystalline as UiO-66, as evidenced by lower intensity and larger width of Bragg reflections for the UiO-66- $\mathrm{NH}_{2}$ product. Tentatively, this might be related to the disorder of the amino group in the crystal structure. The analysis of the behaviour of lattice parameter as function of milling time indicates a similar trend compared with the LAG formation of UiO-66 using DMF (Figure 3b,c). Comparison of the
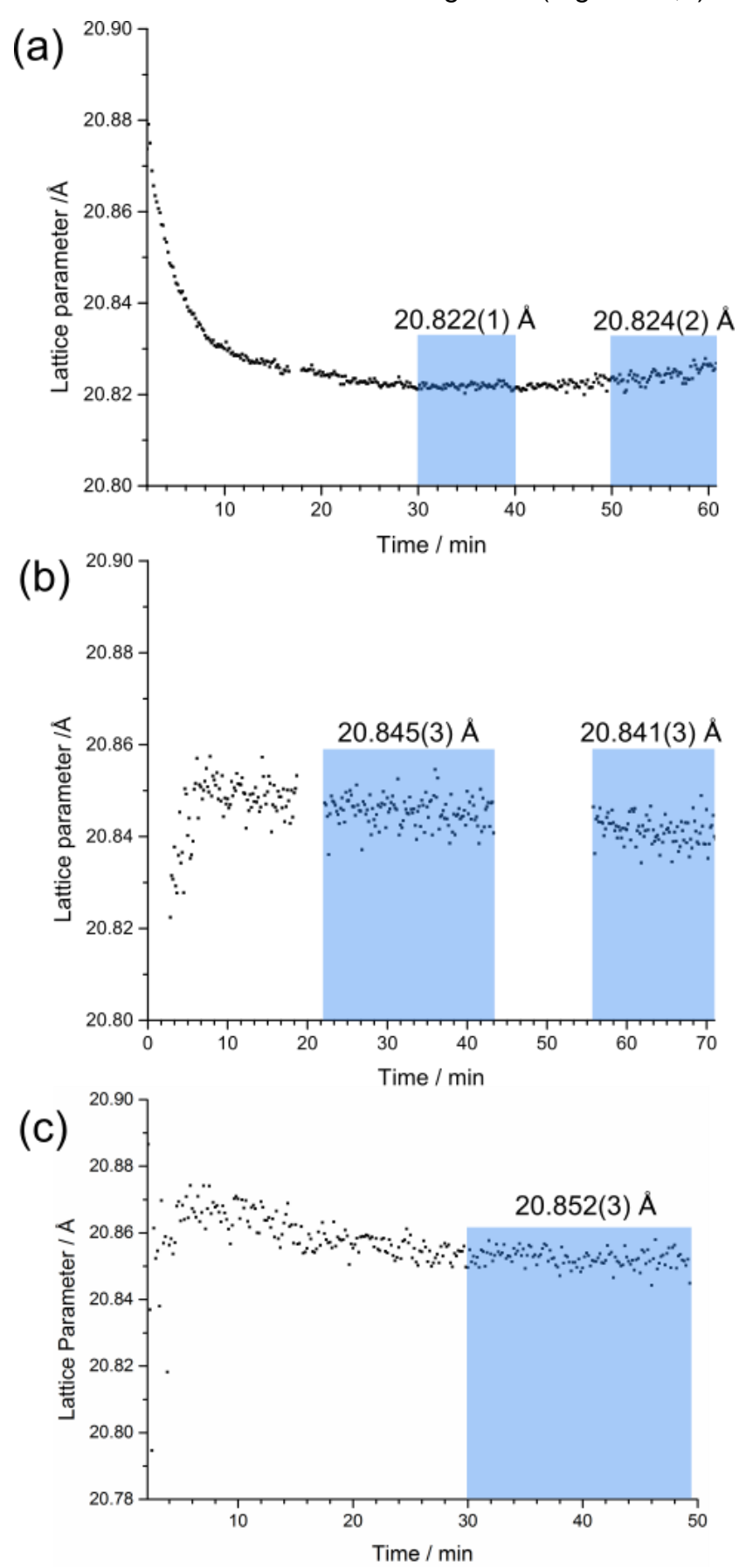

Figure 3. Evolution of the cubic UiO-66 lattice parameter $a$ as a function of $L A G$ reaction time for (a) $\mathrm{MeOH}(\eta=0.78$ $\mu \mathrm{L} / \mathrm{mg}$ ), (b) DMF $(\eta=0.5 \mu \mathrm{L} / \mathrm{mg})$, and (c) UiO-66-NH2 with MeOH $(\eta=0.66 \mu \mathrm{L} / \mathrm{mg})$ as the LAG additive. The shutter of the beamline closed accidentally during the data collection of the reaction using DMF as LAG additive, evidenced by the absence of diffraction signal around 18-22 min and 43-55 min. The first part of both reactions was omitted as it took several scans until UiO-66 started forming. Increase in crystallite size and intensity of the (111) reflection was taken as a measure of when UiO-66 started forming. Lattice parameter a were averaged over the blue areas. 
diffracted signal of both $\mathrm{LAG}$ reactions (using $\mathrm{MeOH}$ or DMF) indicates that the crystallinity is again higher for the reaction involving $\mathrm{MeOH}$ as the LAG additive.

In conclusion, we described the in situ formation of UiO-66 and UiO-66- $\mathrm{NH}_{2}$ monitored by in situ XRPD with a focus on the development of particle size and evolution of the crystal structure in the early stages of milling. Experiments under different conditions revealed a one-step reaction for the formation of UiO-66 structures with different liquid additives (DMF and $\mathrm{MeOH}$ ), using either $\mathrm{H}_{2}$ TPA and $\mathrm{H}_{2}$ TPA-NH $\mathrm{N}_{2}$ as linker. While sequential Rietveld refinement is challenging due to strong X-ray absorption of the steel balls and possible variation of the pore content during the MOF formation, the evolution of crystallite size and integrated peak intensity were found to be a good measure of reaction progress and structural changes upon MOF formation. The synthesis in the presence of methanol leads in general to products with higher crystallinity compared to those in the presence of DMF liquid additive. Further, the reaction using $\mathrm{H}_{2}$ TPA- $\mathrm{NH}_{2}$ instead of $\mathrm{H}_{2}$ TPA as linker further decreased crystallinity, possibly due to the symmetry induced disorder of the 2-amino functional group, as the cubic symmetry in the crystal structure is preserved.

In general, crystallite size and integrated peak intensity of the most prominent reflection are a good measure of reaction progress for porous products. The crystallite size obtained through the analysis of in situ XRPD patterns via the Scherrer equation showed excellent agreement with the particle sizes obtained via SEM analysis. This is potentially important for future applications, where particle size influences the stability of a formed MOF product.

Investigation of lattice parameter as function of reaction time revealed an unexpected behaviour. For the reaction with $\mathrm{MeOH}$ as $\mathrm{LAG}$ additive, a significant contraction of the unit cell was observed. One possible explanation for this behaviour is a gradual release or more homogeneous distribution of guest molecules within the pores. In contrast to this, the lattice parameter during the reaction with DMF as LAG additive remains almost constant. Also, the cell parameters derived from the in situ analysis were found to be slightly larger than most of those reported in the literature. These results suggest that the used zirconium precursor and choice of synthesis route has a strong influence on the final MOF structures and the crystallite size. The scope of the in situ methods is now expanded to processing tools for a green preparation of MOFs with targeted particle sizes.

\section{Acknowledgements}

We thank Ivan Halasz for helpful discussions. This work has been supported by the Croatian Science foundation (Grant 4744). O.K.F. gratefully acknowledges support from the Defense Threat Reduction Agency (HDTRA1-18-1-0003). T. F. acknowledges the financial support of the NSERC Discovery Grant (RGPIN-2017-06467), NSERC Discovery Accelerator award (RGPAS 507837-17), and the NSERC E. W. R. Steacie Memorial Fellowship (SMFSU 507347-17).

\section{References}

[1] I. Halasz, S. A. J. Kimber, P. J. Beldon, A. M. Belenguer, F. Adams, V. Honkimaki, R. C. Nightingale, R. E. Dinnebier and T. Friščić, Nat, Protoc., 2013, 8, 1718-1729,

[2] T. Friščić, I. Halasz, P. J. Beldon, A. M. Belenguer, F. Adams, S. A. J. Kimber, V. Honkimaki and R. E. Dinnebier, Nat. Chem., 2013, 5, 66-73,

[3] K. Užarević, I. Halasz and T. Friščić, J. Phys. Chem. Letter., 2015, 6, 4129-4140.

[4] P. A. Julien, I. Malvestiti and T. Friščić, Beilstein. J. Org. Chem., 2017, 13, 2160-2168.

[5] V. Ban, Y. Sadikin, M. Lange, N. Tumanov, Y. Filinchuk, R. Černý and N. Casati, Anal. Chem., 2017, 89, 13176-13181.

[6] S. Lukin, T. Stolar, M. Tireli, M. V. Blanco, D. Babić, T. Friščić, K. Užarević and I. Halasz, Chem. Eur. J., 2017, 23, 13941-13949.

[7] H. Kulla, M. Wilke, F. Fischer, M. Rollig, C. Maierhofer and F. Emmerling, Chem Commun, 2017, 53, $1664-1667$.

[8] H. Kulla, S. Haferkamp, I. Akhmetova, M. Rollig, C. Maierhofer, K. Rademann and F. Emmerling, Angew Chem Int Edit, 2018, 57, 5930-5933.

[9] L. Batzdorf, F. Fischer, M. Wilke, K. J. Wenzel and F. Emmerling, Angew Chem Int Edit, 2015, 54, $1799-1802$.

[10] N. Tumanova, N. Tumanov, K. Robeyns, F. Fischer, L. Fusaro, F. Morelle, V. Ban, G. Hautier, Y. Filinchuk, J. Wouters, T. Leyssens and F. Emmerling, Cryst Growth Des, 2018, 18, 954-961.

[11] K. Užarević, N. Ferdelji, T. Mrla, P. A. Julien, B. Halasz, T. Friščić and I. Halasz, Chem Sci, 2018, 9, $2525-2532$.

[12] I. Halasz, A. Puskaric, S. A. J. Kimber, P. J. Beldon, A. M. Belenguer, F. Adams, V. Honkimaki, R. E. Dinnebier, B. Patel, W. Jones, V. Štrukil and T. Friščić, Angew Chem Int Edit, 2013, 52, 11538-11541.

[13] F. Delogu and L. Takacs, J. Mater. Sci., 2018, 53, 13331-13342.

[14] D. Hasa, G. S. Rauber, D. Voinovich and W. Jones, Angew. Chem. Int. Ed., 2015, 54, 7371-7375.

[15] D. Hasa, E. Miniussi and W. Jones, Cryst. Growth Des., 2016, 16, 4582-4588. 
[16] J. H. Cavka, S. Jakobsen, U. Olsbye, N. Guillou, C. Lamberti, S. Bordiga and K. P. Lillerud, J Am Chem Soc, 2008, 130, 13850-13851.

[17] M. Kandiah, M. H. Nilsen, S. Usseglio, S. Jakobsen, U. Olsbye, M. Tilset, C. Larabi, E. A. Quadrelli, F. Bonino and K. P. Lillerud, Chem Mater, 2010, 22, 6632-6640.

[18] M. J. Katz, Z. J. Brown, Y. J. Colon, P. W. Siu, K. A. Scheidt, R. Q. Snurr, J. T. Hupp and O. K. Farha, Chem Commun, 2013, 49, 9449-9451.

[19] G. Ryu, M.-K. Kim, M. Park, S. O. Jang, S. H. Kim and H. Jung, Micropor Mesopor Mat, 2019, 274, 9-16.

[20] K. Užarević, T. C. Wang, S. Y. Moon, A. M. Fidelli, J. T. Hupp, O. K. Farha and T. Friščić, Chem Commun, 2016, 52, 2133-2136.

[21] B. Karadeniz, A. J. Howarth, T. Stolar, T. Islamoglu, I. Dejanović, M. Tireli, M. C. Wasson, S.-Y. Moon, O. K. Farha, T. Friščić and K. Užarević, ACS Sustainable Chem. Eng., 2018, 6, 15841-15849.

[22] T. Friščić, S. L. Childs, S. A. A. Rizvi and W. Jones, CrystEngComm., 2009, 11, 418-426.

[23] J. I. Langford and A. J. C. Wilson, J App/ Crystallogr, 1978, 11, 102-113.

[24] A. Lanza, L. S. Germann, M. Fisch, N. Casati and P. Macchi, J Am Chem Soc, 2015, 137, 13072-13078.

[25] F. Millange, C. Serre, N. Guillou, G. Férey and R. I. Walton, Angew Chem Int Ed., 2008, 47, 4100-4105. 